\title{
Note on I-brane Near Horizon PP-wave Background
}

\author{
Rashmi R. Nayak ${ }^{a} *$, Pratap K. Swain $\phi^{\dagger}$ \\ ${ }^{a}$ Centre for Theoretical Studies \\ Indian Institute of Techology Kharagpur, Kharagpur-721 302, India \\ ${ }^{b}$ Department of Physics and Meteorology \\ Indian Institute of Technology Kharagpur, Kharagpur 721302, India
}

\begin{abstract}
We find out a PP-wave spacetime with constant Neveu-Schwarz (NS) three form flux by taking a Penrose limit on the $1+1$ dimensional intersection of two orthogonal stacks of fivebranes in type IIB string theory. We further find out an intersecting (D1-D5)brane solution in this background and analyze its supersymmetry properties by solving the dilatino and gravitino variations explicitly.
\end{abstract}

Keywords: D-brane, PP-wave

\footnotetext{
*Email: rashmi@cts.iitkgp.ernet.in

${ }^{\dagger}$ E-mail: pratap@phy.iitkgp.ernet.in
} 


\section{Introduction}

Study of intersecting brane solutions in supergravities have played an important role in understanding the underlying gauge theories and their dynamics. The so called I-brane background which arises in the $1+1$ dimensional intersection of two orthogonal stacks of five branes in type IIB string theory has been one of the interesting exact string background that has drawn lot of attention in recent past. On one hand from the holography arguments one could expect that the dynamics at the intersection should be holographically related to a $2+1$ dimensional bulk theory, on the other hand there is an enhancement of symmetry in the near horizon geometry. Naively, looking at the theory at the intersection one can expect that this theory should be invariant under $(1+1)$-dimensional Poincare symmetry $I S O(1,1)$, however, as derived in [1, 2] the near horizon geometry describes a $2+1$ dimensional theory with Poincare symmetry $\operatorname{ISO}(2,1)$, and twice as many supercharges one might expect. Furthermore, in [3], these interesting properties were studied from the point of view of D1-brane probe. It was shown that the enhancement of the near horizon geometry has clear impact on the worldvolume dynamics of the D1-brane probe. Further analysis on these and related topics have been discussed in [4, 5, 6, 7].

In the present note we would like to take a Penrose limit on this geometry and look for exact string background. Penrose limit on supergravity D-brane backgrounds has been considered in, for example [8]. Further Penrose limits on backgrounds that correspond to non-local theories has been studied earlier (see for example [9, 10]). For example the Penrose limit on the near horizon geometry of a stack of NS5- branes, was shown to produce a worldsheet theory which is free. Though the corresponding PP-wave background was shown to be supersymmetric, the D-branes seem to break all spacetime superymmetries although they preserve the same worldsheet superymmetries as that of flat space. This issue was resolved in [11] by showing that these supersymmetries do not have zero modes on the worldsheet and hence do not admit local space-time realizations. Hence, unlike the perturbative spectrum, the D-brane spectrum of strings in the linear-dilaton pp-wave is not similar to the flat space case. We would like to further anlayze the fate of these facts in the case of PP-wave background coming from two stacks of intersecting NS5- branes. We find that the Penrose limit on the I-brane geometry gives a background which preserves $1 / 2$ supersymmetries although the parent solution preserves $1 / 4$ spacetime supersymmetries. 
Further, for certain values of parameters this reduces to the well known $H_{6}$ PP-wave background with constant NS-NS 3-form flux. So the sigma model action can be written down directly by following [12], and one can also study classical D-brane solutions in this background. We further present an example of (D1-D5)-brane solution in this background and analyze the spacetime superymmetries. The rest of the paper is organized as follows. In section- 2 we find out a PP-wave background by taking a particular type of Penrose limit on the I-brane background and find that it preserves half of the total spacetime superymmetry. In section-3, we write down the supergravity solution of a class of D1-D5 solution and analyze its supersymmetries. Finally in section-4, we conclude with some comments.

\section{Penrose limit of I-brane background}

We consider the intersection of two stack of NS5-branes on $R^{1,1}$ [1]. We have $k_{1}$ number of NS5- branes extended along $(0,1,2,3,4,5)$ directions and $k_{2}$ number of NS5- branes extended along $(0,1,6,7,8,9)$ directions. For writing down the explicit supergravity background corresponding to the above configuration, let us define

$$
\mathbf{y}=\left(x^{2}, x^{3}, x^{4}, x^{5}\right), \quad \mathbf{z}=\left(x^{6}, x^{7}, x^{8}, x^{9}\right) .
$$

Further assume that $k_{1}$ NS5- branes are localized at the points $z_{n}, n=1, \ldots, k_{1}$, and $k_{2}$ NS5branes are localized at the points $y_{a}, a=1, \ldots, k_{2}$. Every pairs of fivebranes from different sets intersect at different point $\left(y_{a}, z_{n}\right)$. The supergravity background corresponding to this configuration takes the form[1]

$$
\begin{array}{r}
\Phi(z, y)=\Phi_{1}(z)+\Phi_{2}(y), \\
g_{\mu \nu}=\eta_{\mu \nu}, \quad g_{\alpha \beta}=e^{2\left(\phi_{2}-\phi_{2}(\infty)\right)} \delta_{\alpha \beta}, \quad g_{p q}=e^{2\left(\phi_{1}-\phi_{1}(\infty)\right)} \delta_{p q}, \\
H_{\alpha \beta \gamma}=-\epsilon_{\alpha \beta \gamma \delta} \partial^{\delta} \Phi_{2}, \quad H_{p q r}=-\epsilon_{p q r s} \partial^{s} \Phi_{1},
\end{array}
$$

where $(\mu, \nu=0,1),(\alpha, \beta, \gamma, \delta=2,3,4,5)$, and $(p, q, r, s=6,7,8,9)$, and $\Phi$ on the first line of eqn. (2) is the dilaton defined as

$$
e^{2\left(\Phi_{1}-\Phi_{1}(\infty)\right)}=1+\sum_{n=1}^{k_{1}} \frac{l_{s}^{2}}{\left(z-z_{n}\right)^{2}},
$$




$$
e^{2\left(\Phi_{2}-\Phi_{2}(\infty)\right)}=1+\sum_{a=1}^{k_{2}} \frac{l_{s}^{2}}{\left(y-y_{a}\right)^{2}} .
$$

Our goal is to find a PP-wave background of this geometry when $z_{n}=y_{a}=0$. To simplify our notation let us denote

$$
e^{2\left(\Phi_{1}-\Phi_{1}(\infty)\right)}=H_{1}(z), \quad e^{2\left(\Phi_{2}-\Phi_{2}(\infty)\right)}=H_{2}(y)
$$

where for coincident branes the harmonic functions are given by

$$
H_{1}=1+\frac{k_{1} l_{s}^{2}}{z^{2}}, H_{2}=1+\frac{k_{2} l_{s}^{2}}{y^{2}} .
$$

Let us now consider the the near horizon geometry of the above configuration, i.e.

$$
\frac{k_{1} l_{s}^{2}}{z^{2}}>>1, \frac{k_{2} l_{s}^{2}}{y^{2}}>>1
$$

so that the harmonic functions take the following form

$$
H_{1}=\frac{\lambda_{1}}{r_{1}^{2}}, \lambda_{1}=k_{1} l_{s}^{2}, H_{2}=\frac{\lambda_{2}}{r_{2}^{2}}, \lambda_{2}=k_{2} l_{s}^{2} .
$$

Then the metric in eqn. (2) takes the form

$$
d s^{2}=-d t^{2}+\left(d x^{1}\right)^{2}+\frac{\lambda_{1}}{r_{1}^{2}} d r_{1}{ }^{2}+\frac{\lambda_{2}}{r_{2}^{2}} d r_{2}{ }^{2}+\lambda_{1} d \Omega_{1}{ }^{(3)}+\lambda_{2} d \Omega_{2}{ }^{(3)},
$$

where $d \Omega_{1}{ }^{(3)}$ and $d \Omega_{2}{ }^{(3)}$ correspond to the line elements on the unit sphere along $(2,3,4,5)$ and $(6,7,8,9)$ directions respectively. To describe them further we introduce the following coordinates

$$
\begin{aligned}
& x^{2}+i x^{3}=r_{1} \cos \theta_{1} e^{i \phi_{1}}, x^{4}+i x^{5}=r_{1} \cos \theta_{1} e^{i \psi_{1}}, \\
& x^{6}+i x^{7}=r_{2} \cos \theta_{2} e^{i \phi_{2}}, x^{8}+i x^{9}=r_{2} \cos \theta_{2} e^{i \psi_{2}},
\end{aligned}
$$

so that the volume elements on the sphere, and the 2 -form potentials which give the volume forms in eqn. (2) are given by

$$
\begin{aligned}
d \Omega_{1}{ }^{(3)} & =d \theta_{1}^{2}+\sin ^{2} \theta_{1} d \phi_{1}{ }^{2}+\cos ^{2} \theta_{1} d \psi_{1}^{2}, \\
b_{\phi_{1} \psi_{1}} & =\lambda_{1} \cos ^{2} \theta_{1}, \quad 0<\theta_{1}<\frac{\pi}{2}, 0=\phi_{1}, \psi_{1}<2 \pi, \\
d \Omega_{2}{ }^{(3)} & =d \theta_{2}{ }^{2}+\sin ^{2} \theta_{2} d \phi_{2}{ }^{2}+\cos ^{2} \theta_{2} d \psi_{2}{ }^{2}, \\
b_{\phi_{2} \psi_{2}} & =\lambda_{2} \cos ^{2} \theta_{2}, \quad 0<\theta_{2}<\frac{\pi}{2}, 0=\phi_{2}, \psi_{2}<2 \pi,
\end{aligned}
$$


To proceed further, as a first step let us introduce two modes $\rho_{1}$ and $\rho_{2}$ defined by

$$
r_{1}=e^{\frac{\rho_{1}}{\sqrt{\lambda_{1}}}}, r_{2}=e^{\frac{\rho_{2}}{\sqrt{\lambda_{2}}}}
$$

and introduce two modes $r, y$ through

$$
Q r=\frac{\rho_{1}}{\sqrt{\lambda_{1}}}+\frac{\rho_{2}}{\sqrt{\lambda_{2}}}, \quad Q y=\frac{\rho_{1}}{\sqrt{\lambda_{2}}}-\frac{\rho_{2}}{\sqrt{\lambda_{1}}}
$$

where

$$
Q=\frac{1}{\sqrt{\lambda}}, \frac{1}{\lambda}=\frac{1}{\lambda_{1}}+\frac{1}{\lambda_{2}} .
$$

Note that the inverse transformations of (12) take the form

$$
\begin{aligned}
& \rho_{1}=\frac{1}{\sqrt{\lambda_{1}+\lambda_{2}}}\left(\sqrt{\lambda_{1}} y+\sqrt{\lambda_{2}} r\right), \\
& \rho_{2}=\frac{1}{\sqrt{\lambda_{1}+\lambda_{2}}}\left(\sqrt{\lambda_{1}} r-\sqrt{\lambda_{2}} y\right) .
\end{aligned}
$$

Note that this transformation implies that the dilaton is a function of $r$ only, i.e.

$$
\begin{aligned}
\Phi & =\Phi_{1}+\Phi_{2}=\frac{1}{2}\left(H_{1}+H_{2}\right)+\Phi_{1}(\infty)+\Phi_{2}(\infty) \\
& =-\frac{\rho_{1}}{\sqrt{\lambda_{1}}}-\frac{\rho_{2}}{\sqrt{\lambda_{2}}}+\Phi_{0}=-Q r+\Phi_{0} .
\end{aligned}
$$

After doing this substitution, the metric in eqn. (8) and the NS 2-form potentials take the following form

$$
\begin{aligned}
d s^{2} & =-d t^{2}+\left(d x^{1}\right)^{2}+d r^{2}+d y^{2}+\lambda_{1} d \Omega_{1}{ }^{(3)}+\lambda_{2} d \Omega_{2}{ }^{(3)} \\
b_{\phi_{1} \psi_{1}} & =\lambda_{1} \cos ^{2} \theta_{1}, \quad b_{\phi_{2} \psi_{2}}=\lambda_{2} \cos ^{2} \theta_{2} .
\end{aligned}
$$

Now we are ready to take a Penrose limit on this geometry. For the time being let us keep $\lambda_{1}=\lambda_{2}=N^{2}$. One can think that this is an example of asymmetric pp-wave coming from the intersection of two stacks on NS5-branes on a string. Now rescaling $d t=N^{2} d t$, the metric and the NS 2-form potentials become,

$$
\begin{aligned}
d s^{2} & =N^{2}\left(-d t^{2}+\cos ^{2} \theta_{1} d \psi_{1}^{2}+d \theta_{1}^{2}+\sin ^{2} \theta_{1} d \phi_{1}^{2}+\cos ^{2} \theta_{2} d \psi_{2}^{2}+d \theta_{2}^{2}+\sin ^{2} \theta_{2} d \phi_{2}^{2}\right) \\
& +\left(d x^{1}\right)^{2}+d r^{2}+d y^{2} \\
b_{\phi_{1} \psi_{1}} & =N^{2} \cos ^{2} \theta_{1}, \quad b_{\phi_{2} \psi_{2}}=N^{2} \cos ^{2} \theta_{2} .
\end{aligned}
$$


Let us make the following substitution to mix coordinates among the two spheres

$$
\psi_{1}=\cos \alpha \psi_{1}+\sin \alpha \psi_{2}, \quad \psi_{2}=-\sin \alpha \psi_{1}+\cos \alpha \psi_{2}
$$

Then the metric becomes

$$
\begin{aligned}
d s^{2} & =N^{2}\left(-d t^{2}+\left(\cos ^{2} \theta_{1} \cos ^{2} \alpha+\cos ^{2} \theta_{2} \sin ^{2} \alpha\right) d \psi_{1}^{2}+\left(\cos ^{2} \theta_{1} \sin ^{2} \alpha+\cos ^{2} \theta_{2} \cos ^{2} \alpha\right) d \psi_{2}^{2}\right. \\
& \left.+d \theta_{1}^{2}+\sin ^{2} \theta_{1} d \phi_{1}^{2}+d \theta_{2}^{2}+\sin ^{2} \theta_{2} d \phi_{2}^{2}+2 \sin \alpha \cos \alpha\left(\cos ^{2} \theta_{1}-\cos ^{2} \theta_{2}\right) d \psi_{1} d \psi_{2}\right) \\
& +\left(d x^{1}\right)^{2}+d y^{2}+d r^{2} .
\end{aligned}
$$

Lets us now take the Penrose limits as follows [13]:

$$
\begin{aligned}
t & \rightarrow x^{+}+\frac{x^{-}}{N^{2}} \\
\psi_{1} & \rightarrow \frac{1}{\sqrt{2}}\left(x^{+}-\frac{x^{-}}{N^{2}}\right) \\
\psi_{2} & \rightarrow \frac{\psi_{2}}{N}, \theta_{1} \rightarrow \frac{\theta_{1}}{N}, \theta_{2} \rightarrow \frac{\theta_{2}}{N} .
\end{aligned}
$$

and take $N \rightarrow \infty$, the metric becomes

$$
\begin{aligned}
d s^{2} & =-\frac{1}{2}\left(\theta_{1}^{2} \cos ^{2} \alpha+\theta_{2}^{2} \sin ^{2} \alpha\right)\left(d x^{+}\right)^{2}-2 d x^{+} d x^{-} \\
& +d \psi_{2}^{2}+d \theta_{1}^{2}+\theta_{1} d \phi_{1}^{2}+d \theta_{2}^{2}+\theta_{2} d \phi_{2}^{2}+\left(d x^{1}\right)^{2}+d y^{2}+d r^{2} .
\end{aligned}
$$

Note that the dilaton vanishes during this limit. Further let us make the following substitutions

$$
z_{1}=\theta_{1} \cos \phi_{1}, \quad z_{2}=\theta_{1} \sin \phi_{1}, \quad z_{3}=\theta_{2} \cos \phi_{2}, \quad z_{4}=\theta_{2} \sin \phi_{2} .
$$

Now the metric takes the following form (after scaling $x^{+}=2 \mu x^{+}$, and $x^{-}=-x^{-} / 2 \mu$, where $\mu$ is a constant)

$$
d s^{2}=2 d x^{+} d x^{-}-2 \mu^{2}\left[\left(z_{1}^{2}+z_{2}^{2}\right) \cos ^{2} \alpha+\left(z_{3}^{2}+z_{4}^{2}\right) \sin ^{2} \alpha\right]\left(d x^{+}\right)^{2}+\sum_{i=1}^{4} d z_{i}^{2}+\sum_{a=5}^{8} d z_{a}^{2} .
$$


At the same time the NS-NS B-fields become

$$
B_{12}=2 \sqrt{2} \mu x^{+} \cos \alpha, \quad B_{34}=2 \sqrt{2} \mu x^{+} \sin \alpha .
$$

which gives the constant 3-form NS-NS flux

$$
H_{+12}=2 \sqrt{2} \mu \cos \alpha, \quad H_{+34}=2 \sqrt{2} \mu \sin \alpha .
$$

Let us discuss this background in a bit more detail. Now for $\sin \alpha=\cos \alpha=\frac{1}{\sqrt{2}}$, this is the well known $H_{6} \mathrm{PP}$-wave background coming from the $A d S_{3} \times S^{3}$ geometry. For $\alpha=0$, (or $\pi / 2$ ), this is the background obtained by taking the near horizon pp-wave limit of a stack of NS5-brane [9]. Let us now look at the supersymmetry this background preserve. It is well known that the I-brane background preserves $1 / 4$ superymmetries. One can check that this background above preserves $1 / 2$ of the total spacetime superymmetry after imposing the condition

$$
\Gamma^{\hat{+}} \epsilon_{ \pm}=0
$$

The sigma model action can be written down directly following [12. Few comments are in order now. In fact one can start with a background which is the $S$-dual of the one that is considered here, namely two stacks of $D 5$-branes intersecting over a line and take a Penrose limit. One will get the same background metric as above but the NS-NS flux will be replaced by the Ramond-Ramond 3-form flux. Furthermore, one can also uplift this to the $M 5-M 5^{\prime}$ intersecting over a 2-plane and take the Penrose limit of the geometry. The $R-R$ three form flux will be replaced by $R-R$ four form flux. Similar solutions are discussed in [14, 15] earlier.

In fact one could further notice that this background with a light like linear dilaton is also a solution of type IIB supergravity equations of motion. The metric, null dilaton and the 3 -from flux in this case is given by

$$
\begin{aligned}
& d s^{2}=2 d x^{+} d x^{-}-2 \mu^{2}\left(x^{+}\right)\left[\left(z_{1}^{2}+z_{2}^{2}\right) \cos ^{2} \alpha+\left(z_{3}^{2}+z_{4}^{2}\right) \sin ^{2} \alpha\right]\left(d x^{+}\right)^{2}++\sum_{a=1}^{8} d z_{a}^{2}, \\
& H_{+12}=2 \sqrt{2} \mu\left(x^{+}\right) \cos \alpha, \quad H_{+34}=2 \sqrt{2} \mu\left(x^{+}\right) \sin \alpha, \Phi=\Phi\left(x^{+}\right)
\end{aligned}
$$




\section{$3 \quad$ Intersecting D-brane solutions}

We now write down the supergravity solution for intersecting (D1-D5)-branes in the background (23) and (25). The metric, dilaton and the field strengths of such a configuration is give by

$$
\begin{aligned}
d s^{2} & =\left(f_{1} f_{5}\right)^{-1 / 2}\left(2 d x^{+} d x^{-}-2 \mu^{2}\left[\left(z_{1}^{2}+z_{2}^{2}\right) \cos ^{2} \alpha+\left(z_{3}^{2}+z_{4}^{2}\right) \sin ^{2} \alpha\right]\left(d x^{+}\right)^{2}\right) \\
& +\left(\frac{f_{1}}{f_{5}}\right)^{1 / 2} \sum_{a=5}^{8} d z_{a}^{2}+\left(f_{1} f_{5}\right)^{1 / 2} \sum_{i=1}^{4} d z_{i}^{2}, \quad e^{2 \phi}=\left(\frac{f_{1}}{f_{5}}\right)^{1 / 2} \\
H_{+12} & =2 \sqrt{2} \mu \cos \alpha, \quad H_{+34}=2 \sqrt{2} \mu \sin \alpha, \\
F_{+-i} & =\partial_{i} f_{1}^{-1}, \quad F_{i j k}=\epsilon_{i j k l} \partial_{l} f_{5}, \quad f_{1,5}=1+\frac{Q_{1,5}}{r^{2}}
\end{aligned}
$$

where $f_{1,5}$ are the harmonic functions of the D1 and D5-brane in the transverse 4-space respectively. The above (D1-D5)-brane configuration solves all type IIB field equations. One can notice that for $\cos \alpha=\sin \alpha=\frac{1}{\sqrt{2}}$, the above solution reduces to a (D1-D5)-brane solution in a PP-wave background coming from pure $A d S_{3} \times S^{3}$ with NS-NS flux [18]. Further intersecting brane solutions can be found out by applying $T$-dualities along the $z^{a}$ directions. One can further check that by putting $f_{1}=1$, the above solution reduces to a D5-brane lying along $(+,-, 5,6,7,8)$ directions and by putting $f_{5}=1$, this reduces to a localized D1-brane lying along $(+,-)$ directions.

Now let us check the amount of unbroken supersymmetries the above (D1-D5)-brane solution preserves. The supersymmetry variations of dilatino and gravitino fields of type IIB supergravity in the string frame are given by [16, 17],

$$
\begin{aligned}
\delta \lambda_{ \pm} & =\frac{1}{2}\left(\Gamma^{\mu} \partial_{\mu} \phi \mp \frac{1}{12} \Gamma^{\mu \nu \rho} H_{\mu \nu \rho}\right) \epsilon_{ \pm}+\frac{1}{2} e^{\phi}\left( \pm \Gamma^{\mu} F_{\mu}^{(1)}+\frac{1}{12} \Gamma^{\mu \nu \rho} F_{\mu \nu \rho}^{(3)}\right) \epsilon_{\mp} \\
\delta \Psi_{\mu}^{ \pm} & =\left[\partial_{\mu}+\frac{1}{4}\left(w_{\mu \hat{A} \hat{B}} \mp \frac{1}{2} H_{\mu \hat{A} \hat{B}}\right) \Gamma^{\hat{A} \hat{B}}\right] \epsilon_{ \pm} \\
& +\frac{1}{8} e^{\phi}\left[\mp \Gamma^{\lambda} F_{\lambda}^{(1)}-\frac{1}{3 !} \Gamma^{\lambda \nu \rho} F_{\lambda \nu \rho}^{(3)} \mp \frac{1}{2.5 !} \Gamma^{\lambda \nu \rho \alpha \beta} F_{\lambda \nu \rho \alpha \beta}^{(5)}\right] \Gamma_{\mu} \epsilon_{\mp},
\end{aligned}
$$

where $\mu, \nu, \rho, \lambda$ are ten dimensional space-time indices, and hated indices refer to the Lorentz frame. Note that for the background (23) - (25) , the vanishing of the above supersymmetry variations leads to the Killing spinors,

$$
\epsilon_{ \pm}^{(b g)}=e^{ \pm \frac{\mu}{\sqrt{2}} x^{+}\left(\Gamma^{\hat{1} \hat{2}} \cos \alpha+\Gamma^{\hat{3} \hat{4}} \sin \alpha\right)} \epsilon_{ \pm}^{(0)}, \quad \Gamma^{\hat{+}} \epsilon_{ \pm}^{(0)}=0
$$


where $\epsilon_{ \pm}^{(0)}$ are constant spinors. Now let us solve the above dilatino and gravitino varioations for the (D1-D5)-brane solution presented in (28). First the dilatino variation gives the following condition on the spinors.

$$
\begin{gathered}
\Gamma^{\hat{i}} \epsilon_{ \pm}-\Gamma^{\hat{+} \hat{-} \hat{i}} \epsilon_{\mp}=0, \\
\Gamma^{\hat{i}} \epsilon_{ \pm}+\frac{1}{3 !} \epsilon_{\hat{i} \hat{j} \hat{k} \hat{l}} \Gamma^{\hat{j} \hat{k} \hat{l}} \epsilon_{\mp}=0, \\
\left(\Gamma^{\hat{+} \hat{1} \hat{2}}+\tan \alpha \Gamma^{\hat{+3} \hat{4} \hat{4}}\right) \epsilon_{\mp}=0 .
\end{gathered}
$$

The first two are the usual D1-brane and D5-brane supersymmetry condition even in the flat space. The third condition is related to the property of this particular PP-wave spacetime. We will come back to this after solving the gravitino variations more carefully. On the other hand, solving gravitino variations gives the following conditions on the spinors

$$
\begin{aligned}
\delta \psi_{+}^{ \pm} & \equiv \partial_{+} \epsilon_{ \pm} \mp \frac{\mu}{\sqrt{2}}\left(f_{1} f_{5}\right)^{-1 / 2}\left(\Gamma^{\hat{1} \hat{2}} \cos \alpha+\Gamma^{\hat{3} \hat{4}} \sin \alpha\right) \epsilon_{ \pm}=0, \quad \delta \psi_{-}^{ \pm} \equiv \partial_{-} \epsilon_{ \pm}=0 \\
\delta \psi_{i}^{ \pm} & \equiv \partial_{+} \epsilon_{ \pm}+\frac{1}{8}\left[\frac{f_{1, i}}{f_{1}}+\frac{f_{5, i}}{f_{5}}\right] \epsilon_{ \pm}=0, \quad \delta \psi_{a}^{ \pm} \equiv \partial_{a} \epsilon_{ \pm}=0
\end{aligned}
$$

where in writing down the above variations, we have made use of the condition

$$
\Gamma^{\hat{+}} \epsilon_{ \pm}=0
$$

and the D-brane supersymmetry conditions written in (32) and (33). In what follows, we will discuss the supersymmetry for this brane solution for the parameter $\sin ^{2} \alpha=\cos ^{2} \alpha=$ $1 / 2$, as it is more evident. One can check that imposing conditions (36), (32), and (33) together with

$$
\left(1-\Gamma^{\hat{1} \hat{2} \hat{3} \hat{4}}\right) \epsilon_{ \pm}=0
$$

all the variations are satisfied. Hence the (D1-D5)-brane solution presented above satisfies $1 / 8$ of the total spacetime supersymmetries, as there are only three independent conditions to be imposed. Similar supersymmetry analysis for intersecting branes with constant NSNS flux has been presented in [18]. The worldsheet superymmetries can be analyzed by looking at [19]. Hence we skip the details here. 


\section{Conclusions}

In this paper we have found out a PP-wave background with constant three form flux by taking a Penrose limit of a $1+1$ dimensional orthogonal intersection of two stack of NS5brans in supergravities. This background is shown to be $1 / 2$ superymmetric. We further find out an intersecting (D1-D5)-brane solution in this background and study its spacetime superymmetry properties by solving the dilatino and gravitino variations explicitly. We have shown that unlike the PP-wave background coming from the near horizon Penrose limit of a single stack of NS5-branes, here the nonperturbative spectrum is similar to the flat space counterpart.

\section{References}

[1] N. Itzhaki, D. Kutasov and N. Seiberg, "I-brane dynamics," JHEP 0601, 119 (2006) arXiv:hep-th/0508025.

[2] H. Lin and J. M. Maldacena, "Fivebranes from gauge theory," Phys. Rev. D 74, 084014 (2006) arXiv:hep-th/0509235.

[3] J. Kluson, "Dynamics of D1-brane in I-brane background," JHEP 0512, 016 (2005) arXiv:hep-th/0510243.

[4] J. Kluson, "Note about static D1-brane in I-brane background," JHEP 0601, 045 (2006) arXiv:hep-th/0511304.

[5] L. Y. Hung, "Intersecting D7-branes, I5-branes and conifolds," JHEP 0703, 071 (2007) arXiv:hep-th/0612070.

[6] L. Y. Hung, "Comments on I1-branes," JHEP $0705 \quad$ (2007) 076 arXiv:hep-th/0612207].

[7] J. Kluson, "Fundamental String and D1-brane in I-brane Background," JHEP 0801, 045 (2008) arXiv:0711.4219 [hep-th]].

[8] M. Blau, J. M. Figueroa-O'Farrill and G. Papadopoulos, "Penrose limits, supergravity and brane dynamics," Class. Quant. Grav. 19, 4753 (2002) arXiv:hep-th/0202111. 
[9] V. E. Hubeny, M. Rangamani and E. P. Verlinde, "Penrose limits and non-local theories," JHEP 0210, 020 (2002) arXiv:hep-th/0205258.

[10] M. Alishahiha and A. Kumar, "PP-waves from nonlocal theories," JHEP 0209, 031 (2002) arXiv:hep-th/0207257.

[11] S. F. Hassan, R. R. Nayak and K. L. Panigrahi, "D-branes in the NS5 near-horizon pp-wave background," arXiv:hep-th/0312224.

[12] J. G. Russo and A. A. Tseytlin, "On solvable models of type IIB superstring in NS-NS and R-R plane wave JHEP 0204, 021 (2002) [arXiv:hep-th/0202179].

[13] H. Lu and J. F. Vazquez-Poritz, "Penrose limits of non-standard brane intersections," Class. Quant. Grav. 19, 4059 (2002) arXiv:hep-th/0204001.

[14] M. Cvetic, H. Lu and C. N. Pope, "M-theory pp-waves, Penrose limits and supernumerary supersymmetries," Nucl. Phys. B 644, 65 (2002) arXiv:hep-th/0203229].

[15] M. Cvetic, H. Lu and C. N. Pope, "Penrose limits, pp-waves and deformed M2branes," Phys. Rev. D 69, 046003 (2004) arXiv:hep-th/0203082.

[16] J. H. Schwarz, "Covariant Field Equations Of Chiral N=2 D=10 Supergravity," Nucl. Phys. B 226, 269 (1983).

[17] S. F. Hassan, "T-duality, space-time spinors and R-R fields in curved backgrounds," Nucl. Phys. B 568, 145 (2000) arXiv:hep-th/9907152].

[18] A. Biswas, A. Kumar and K. L. Panigrahi, "p - p' branes in pp-wave background," Phys. Rev. D 66, 126002 (2002) arXiv:hep-th/0208042.

[19] Y. Michishita, "D-branes in NSNS and RR pp-wave backgrounds and S-duality," JHEP 0210, 048 (2002) arXiv:hep-th/0206131. 